\title{
Neural Network for Complex Systems: Theory and Applications
}

\author{
Chenguang Yang $\mathbb{D}^{1},{ }^{1}$ Jing $\mathrm{Na}^{2}{ }^{2}$ Guang $\mathrm{Li},{ }^{3}$ Yanan $\mathrm{Li}^{4}{ }^{4}$ and Junpei Zhong ${ }^{5}$ \\ ${ }^{1}$ College of Automation Science and Engineering, South China University of Technology, Guangzhou, China \\ ${ }^{2}$ Faculty of Mechanical and Electrical Engineering, Kunming University of Science and Technology, Kunming, China \\ ${ }^{3}$ School of Engineering and Materials Science, Queen Mary University of London, London, UK \\ ${ }^{4}$ Department of Engineering and Design, University of Sussex, Brighton, UK \\ ${ }^{5}$ National Institute of Advanced Industrial Science and Technology, Tokyo, Japan \\ Correspondence should be addressed to Chenguang Yang; cyang@ieee.org
}

Received 7 March 2018; Accepted 13 March 2018; Published 8 May 2018

Copyright (c) 2018 Chenguang Yang et al. This is an open access article distributed under the Creative Commons Attribution License, which permits unrestricted use, distribution, and reproduction in any medium, provided the original work is properly cited.

Over the last few decades, neural network (NN) has been successfully employed in a wide range of applications as the joint effort from both industrial and academic communities. With its powerful approximation ability, $\mathrm{NN}$ has been utilized into many promising fields, such as modelling and identification of complex and nonlinear systems and optimization and automatic control. The components integrated with a complex system may interact with each other and bring difficulties to the control. Hence, research of the complex system is becoming increasingly important in both the natural and social sciences. In this special issue, we bring together a number of important works. The contributors have investigated various applications of a wide range of complex systems such as robots and transportation systems and have also achieved a number of advances in fundamental theoretical studies.

In the field of robotics, Y. Jiang et al. investigated and surveyed the state of the art on NN based robot learning and control applications. In this work, the authors summarized recent progresses in neural networks and their applications in robots, for example, $\mathrm{NN}$ based robot manipulator control, NN based human robot interaction, and NN based behaviour recognition and generation. G. Gao et al. proposed a modelling and error compensation method for articulated arm coordinate measuring machine (AACMM) based on BP neural network, which shows that $97 \%$ error of the AACMM can be removed after compensation. Z. Guo et al. derived a multi-input multi-output complex nonlinear dynamic model to fully describe serial variable stiffness actuators (SVSAs) and proposed an NN-based adaptive control strategy based on feedback linearization to handle system uncertainties. R. Mei and C. Yu presented an adaptive neural output feedback control scheme for uncertain robot manipulators with input saturation using the radial basis function neural network (RBFNN) and disturbance observer. M. Wang et al. developed a dynamic learning method for an uncertain $n$-link robot with unknown system dynamics and achieved predefined performance attributes on the link angular position and velocity tracking errors. In another work, $M$. Wang et al. focused on neural learning from the adaptive neural control (ANC) for a class of flexible joint manipulator with unknown dynamics under the output tracking error constraint. X. Zhang et al. presented a skill learning method by perceptionaction integration strategy from the perspective of hierarchical temporal memory (HTM) theory. B. Xu and P. Zhang proposed the sliding mode control while the adaptive design was developed using neural networks $(\mathrm{NNs})$ and disturbance observer (DOB) with novel update laws for $\mathrm{NN}$ and DOB. L. Wang et al. proposed a three-layer perception framework based on transfer learning which improved the environmental perception ability of mobile robots during semantic navigation.

On complex system control issues, L. Song et al. used the federal Kalman Filter (FKF) based on NN in the velocity and attitude matching of transfer alignment. L. Ma and D. Li proposed an adaptive NN control approach for a directcurrent (DC) system with full state constraints. J. Ding used the hierarchical identification principle to present a 
hierarchical gradient based iterative (H-GI) algorithm and a hierarchical least square based iterative (H-LSI) algorithm. B. $\mathrm{Xu}$ and $\mathrm{P}$. Zhang investigated an adaptive neural sliding mode controller for MEMS gyroscopes with minimal-learningparameter technique. C. Li et al. proposed a combined system modelling approach to approximate the actual vehicle system. S.-M. Lu and D.-J. Li addressed an adaptive NN control problem for a class of nonlinear hydraulic servosystem with time-varying state constraints. Y. Yin et al. proposed a novel NN sliding mode control based on a multicommunity bidirectional drive collaborative search algorithm (M-CBDCS) to design a flight controller for performing the attitude tracking control of a quad tilt rotors aircraft (QTRA). Z. Li et al. proposed a general framework of a nonlinear recurrent NN for online solving generalized linear matrix equation (GLME) with global convergence property. S. Tronci and R. Baratti presented a gain-scheduling design technique that relies upon neural models to approximate plant behaviour. J.-E. Zhang showed that every subnetwork of a class of coupled fractionalorder neural networks consisting of $N$ identical subnetworks can have $(r+1) n$ locally Mittag-Leer stable equilibria. $\mathrm{Z}$. Mao and F. Zhao studied the effect of different dimensionless plate lengths and damping values on the UMP in the case of constant flow rate.

In the field of transportation, L. Wang et al. constructed a simulation platform of urban traffic integration and proposed a feasible data analysis, learning and parameter calibration method based on RBFNN. S. Chen et al. developed a multiconstrained network intensive vehicle routing algorithm based on an adaptive ant colony algorithm. D. Gong et al. studied the pinning synchronous problem for complex networks with interval delays and proposed a series of useful theories. Y. E. Shao et al. proposed a two-NN based scheme to enhance the accurate identification rate (AIR) for MCCPs by performing dimension reduction on disturbance categories.

On the complex systems of the nature, biology, and the human body, R. Rao and S. Zhong investigated the stochastically exponential stability of reaction-diffusion impulsive stochastic cellular neural networks (CNN). S. Rómoli et al. proposed an online state estimator based on an RBFNN to solve the question of the lack of online information on some bioprocess variables and the presence of modelling and parametric uncertainties. $H$. Zhou et al. proposed a large-scale neural circuit model and showed that parallel excitatory and inhibitory pathways underlie the learned neural responses across multiple brain regions. Z. Xiao et al. proposed a BP $\mathrm{NN}$ for the prediction of aquaculture dissolved oxygen. C. S. Chin et al. used the transfer learning and deep convolutional neural network $(\mathrm{CNN})$ to perform image recognition on the fouling image by classifying the detected fouling species and the density of fouling on the surface.

On the classification, optimization, and diagnostic issues of a complex system, J. Wang et al. used the finite-time stable control method with RBF neural network to suppress the structural vibration. Á. Morera et al. described an experimental study on the suitability of deep NN to three automatic demographic problems. W. Tian et al. presented an optimization method for the design of the layout of an autonomous underwater vehicles (AUV) fleet to minimize the drag force. Q. Wang et al. proposed semiactive nonsmooth control algorithm with deep learning to suppress harmful effect for building the structure by a surface motion. W. Liu et al. used a RBFNN model to analyse the impacts of six equities on the acquisition of university spin-outs (USOs). J. Ma et al. introduced the multikernel function and costsensitive mechanism to construct the fault diagnosis model of check valve based on the multikernel cost-sensitive extreme learning machine (MKL-CS-ELM).

The selected papers in the special issue could not cover all the recent advances of the NN technology for complex systems, yet they present the state-of-the-art progress in this area. We hope these valuable papers may enrich the knowledge in the complex systems community and provide guidance to the readers who are interested in this topic.

\section{Acknowledgments}

The guest editors would like to acknowledge and appreciate the authors and the reviewers for their contribution towards the success of this special issue.

Chenguang Yang
Jing Na
Guang Li
Yanan Li
Junpei Zhong




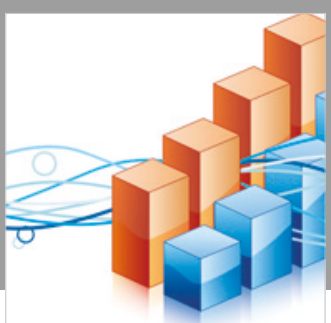

Advances in

Operations Research

\section{-n-m}
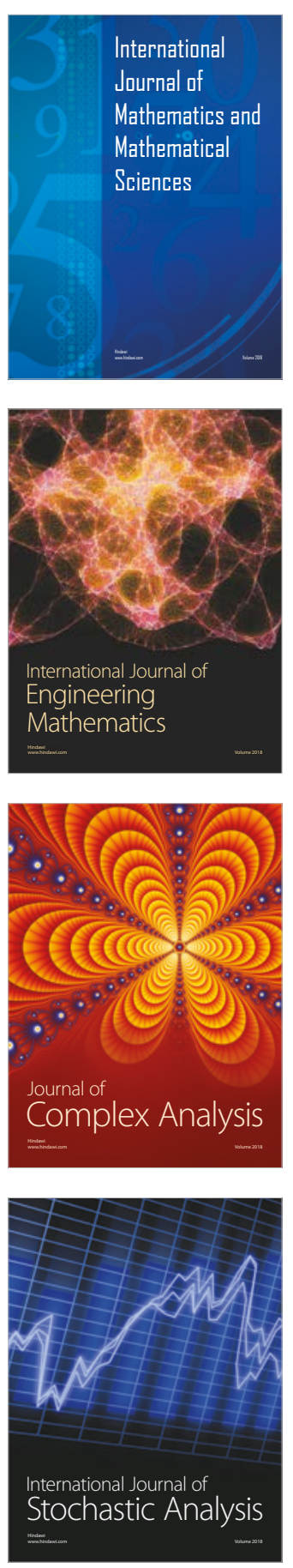
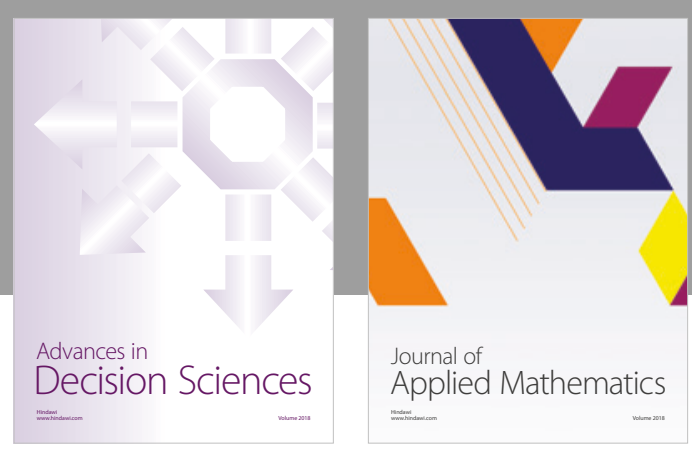

Journal of

Applied Mathematics
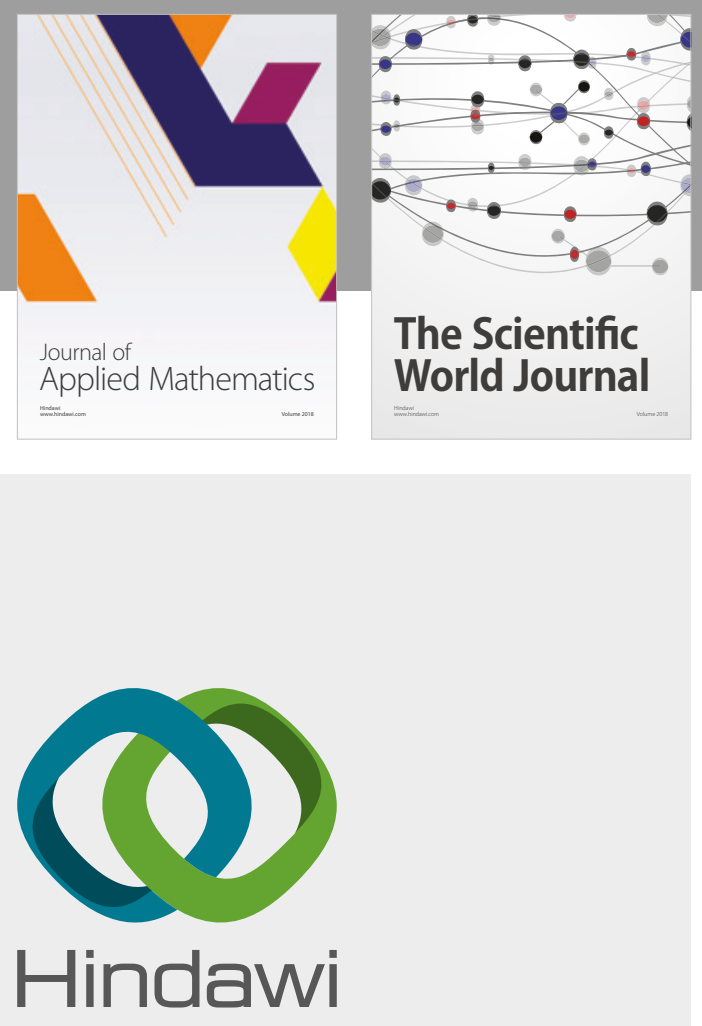

Submit your manuscripts at

www.hindawi.com

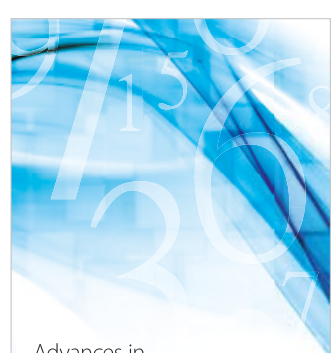

Advances in
Numerical Analysis
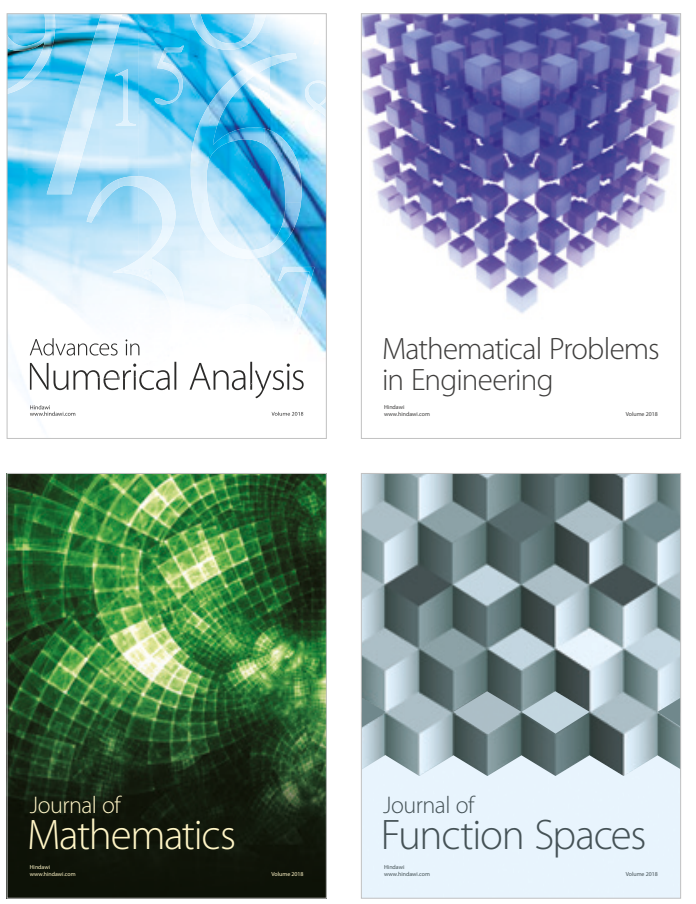

Mathematical Problems in Engineering

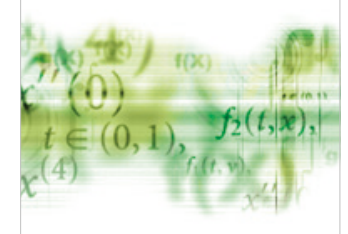

International Journal of

Differential Equations

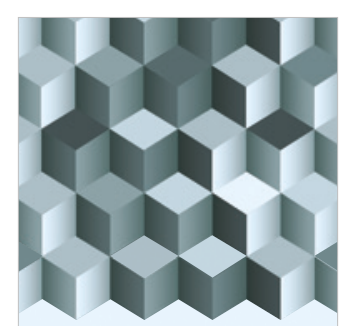

Journal of

Function Spaces
The Scientific

World Journal

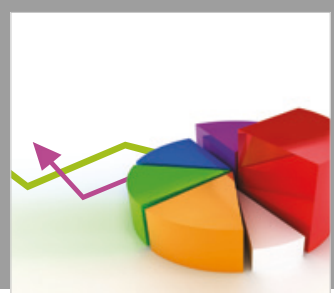

Journal of

Probability and Statistics
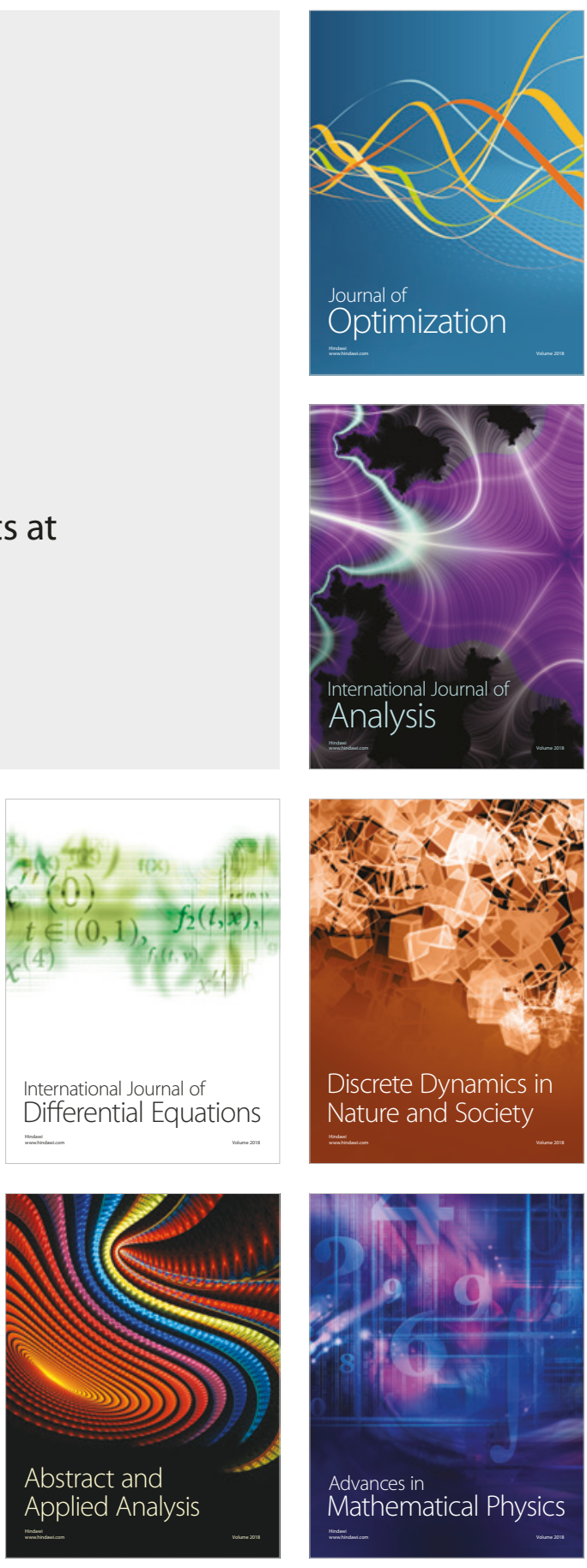